\title{
Atuação do fisioterapeuta neurofuncional no paciente com Doença de Parkinson: uma
}

\section{revisão narrativa}

Performance of the neurofunctional physiotherapist in the patient with Parkinson's Disease: a narrative review

Desempeño del fisioterapeuta neurofuncional en el paciente con Enfermedad de Parkinson: una revisión narrativa

Recebido: 04/01/2022 | Revisado: 09/01/2022 |Aceito: 14/01/2022 | Publicado: 18/01/2022

\author{
Sheyla da Silva Santos \\ ORCID:https://orcid.org/0000-0002-0296-9590 \\ Centro Universitário Mário Pontes Jucá, Brasil \\ E-mail: sheylafisiosheyla@gmail.com \\ Thauan Narciso de Lima Ferro \\ ORCID: https://orcid.org/0000-0002-0174-6467 \\ Centro Universitário Mário Pontes Jucá, Brasil \\ E-mail: thauanferro@hotmail.com
}

\begin{abstract}
Resumo
Segundo a portaria ${ }^{\circ}$ 228, de 10 de maio de 2010 do Ministério da Saúde, a doença de Parkinson (DP), antes descrita por James Parkinson em 1817, é uma das doenças neurológicas mais comuns e intrigantes da atualidade. A patologia é caracterizada por quatro sinais clínicos essenciais: Tremor de repouso, rigidez, bradicinesia e instabilidade postural. O presente estudo avaliou a importância da atuação da fisioterapia na doença de Parkinson, buscando traçar os principais pontos das intervenções realizadas por estes profissionais. O trabalho visou a fazer uma revisão bibliográfica e atualização sobre a atuação da fisioterapia na Doença de Parkinson. Assim foram consultados o Pubmed, o Scielo e o Lilacs com as palavras fisioterapia X doença de Parkinson, tanto artigos em Português quanto em Inglês. A fisioterapia é empregada como tratamento adjunto aos medicamentos ou a cirurgia utilizada na Doença de Parkinson. Essa revisão bibliográfica mostrou que há benefícios de todas as intervenções relacionadas a fisioterapia convencional e também com a fisioterapia neurológica.
\end{abstract}

Palavras-chave: Doença de Parkinson; Fisioterapia neurofuncional; Disfunções neurológicas.

\begin{abstract}
According to Ordinance No. 228, of May 10, 2010, from the Ministry of Health, Parkinson's disease (PD), previously described by James Parkinson in 1817, is one of the most common and intriguing neurological diseases today. The pathology is characterized by four essential clinical signs: tremor at rest, stiffness, bradykinesia and postural instability. The present study evaluated the importance of the performance of physiotherapy in Parkinson's disease, seeking to outline the main points of the interventions performed by these professionals. The work aimed to make a bibliographic review and update on the performance of physiotherapy in Parkinson's Disease. Thus, Pubmed, Scielo and Lilacs were consulted with the words physiotherapy X Parkinson's disease, both articles in Portuguese and in English. Physiotherapy is used as an adjunct treatment to medications or the surgery used in Parkinson's Disease. This bibliographic review showed that there are benefits from all interventions related to conventional physiotherapy and also to neurological physiotherapy.

Keywords: Parkinson's disease; Neurofunctional physiotherapy; Neurological dysfunctions.
\end{abstract}

\section{Resumen}

Según la Ordenanza No. 228, del 10 de mayo de 2010 del Ministerio de Salud, la enfermedad de Parkinson (EP), descrita anteriormente por James Parkinson en 1817, es una de las enfermedades neurológicas más comunes e intrigantes en la actualidad. La patología se caracteriza por cuatro signos clínicos esenciales: temblor en reposo, rigidez, bradicinesia e inestabilidad postural. El presente estudio evaluó la importancia del papel de la fisioterapia en la enfermedad de Parkinson, buscando esbozar los principales puntos de las intervenciones realizadas por estos profesionales. El trabajo tuvo como objetivo realizar una revisión de la literatura y una actualización sobre el papel de la 
fisioterapia en la Enfermedad de Parkinson. Así, Pubmed, Scielo y Lilacs fueron consultados con las palabras fisioterapia $\mathrm{X}$ Enfermedad de Parkinson, ambos artículos en portugués y en inglés. La fisioterapia se usa como tratamiento adjunto a los medicamentos o la cirugía que se usan en la enfermedad de Parkinson. Esta revisión de la literatura mostró que existen beneficios de todas las intervenciones relacionadas con la fisioterapia convencional y también con la fisioterapia neurológica.

Palabras clave: Enfermedad de Parkinson; Fisioterapia neurofuncional; Desórdenes neurológicos.

\section{Introdução}

Segundo a portaria $\mathrm{n}^{\circ}$ 228, de 10 de maio de 2010 do Ministério da Saúde, a doença de Parkinson (DP), antes descrita por James Parkinson em 1817, é uma das doenças neurológicas mais comuns e intrigantes da atualidade. É uma doença que perpetua todo o mundo, e atinge todos os grupos étnicos e classes sócio-econômicas.

A doença de Parkinson é uma disfunção neurológica progressiva e é causada pela degeneração dos neurônios da substância negra compacta do mesencéfalo - uma pequena área do tronco encefálico (Suzuki \& De Carvalho, 2021).

A doença de Parkinson (DP) se caracteriza como uma doença crônica e neurodegenerativa, com incidência na população acima de 65 anos de 1 a $2 \%$ em todo o mundo e prevalência no Brasil de 3\% (Navarro, 2015). O Parkinson é a segunda doença neurodegenerativa mais comum, no mundo (Ferreira, 2020), entre indivíduos maiores de 60 anos (Da Costa Silva, 2011), com frequência maior entre os homens (Ferreira, 2020). Nas últimas décadas, o crescimento da população de idosos fez com que aumentasse o impacto da DP em termos de saúde pública (Souza, 2002).

Primeiramente citada por James Parkinson, a doença de Parkinson é um dos distúrbios de movimento que mais acomete os idosos. Para Parkinson (1817), autor cujo nome deu à patologia, a definição mais cabível para esta patologia é de que se trata de uma doença de evolução lenta caracterizada pela presença de movimentos involuntários trêmulos, redução da força muscular, flexão do tronco e com alteração da marcha, o que levaria a quedas frequentes, tendo os sentidos e intelecto inalterados. Do ponto de vista clínico, a doença de Parkinson também se caracteriza pelabradicinesia e insuficiência autônoma.

Segundo Tieve (1998), a patologia é caracterizada por quatro sinais clínicos essenciais: Tremor de repouso, rigidez, bradicinesia e instabilidade postural. O mesmo acrescenta que a patologia apresenta como manifestações secundárias incoordenação motora, micrografia, embaçamento da visão, disartria, edema, sialorréia, face em máscara, deformidade de mão e pé, distonia, escoliose, cifose, demência, depressão.

A fisiopatologia dos distúrbios do movimento é atribuída a uma disfunção nos núcleos da base. Acredita-se que estes núcleos sejam responsáveis pela modulação e facilitação de vários programas motores e cognitivos de origem cortical (Souza, 2002). O sistema extrapiramidal pode promover ou inibir o movimento, dependendo da inervação dopaminérgica do estriado (Hauser \& Zesiewicz, 2001).

O sistema dopaminérgico junto com os neurônios de melanina sofre despigmentação. Desta forma, subentende-se que quanto mais clara a substância negra, maior é a perda de dopamina (Teive, 2003). A Doença de Parkinson é caracterizada pela degeneração dos neurônios dopaminérgicos localizados na parte compacta da substância negra, que leva a diminuição na produção da dopamina (Da Costa Silva, 2011).

Associado a essa despigmentação, segundo Faria (2006), tem-se a depleção do neurotransmissor dopamina que resulta da degeneração de neurônios dopaminérgicos da substância negra que se projetam para o estriado. Onde, acrescenta o estudo de Faria (2006), são críticos para o controle do processamento da informação pelos gânglios da base, reduzindo a atividade das áreas motoras do córtex cerebral, desencadeando a diminuição dos movimentos voluntários. 
Apresenta uma etiologia idiopática, porém acredita-se que os seus surgimentos provem de fatores ambientais e genéticos, podendo interagir e contribuir para o desenvolvimento neurodegenerativo da DP (Pereira, 2010).

Embora as manifestações clínicas clássicas da DP sejam facilmente reconhecíveis, traduzem um processo degenerativo de alguns anos. O envelhecimento de áreas externas ao sistema dopaminérgico nigroestriatal, aliado ao envelhecimento do sistema nervoso autônomo periférico são responsáveis por múltiplos sinais e sintomas não-motores (Suzuki \& De Carvalho, 2021). As manifestações autonômicas da DP são disfagia, disfunções

cardiovasculares, urogenital, termorregulatória, respiratória e gastrointestinal.

Como em toda patologia de caráter degenerativo, esses comprometimentos causam declínio funcional, dificultando, desta maneira, a execução das atividades de vida diária (AVD), podendo, ainda, resultarem, segundo Da Costa Silva (2011), diminuição da independência física e da qualidade de vida.

O acometimento do sistema nervoso periférico pela presença de corpos de Lewy no sistema nervoso autonômico em pacientes com a DP avançada poderia explicar alterações urinárias esfincterianas e alguns casos de hiporreflexia do músculo detrusor da bexiga (Araki \& Kuno, 2000).

A doença de Parkinson ou parkinsonismo idiopático é uma doença com características clínicas e uma evolução natural bem conhecida, mas os exames de neuroimagem são inespecíficos e não há um marcador biológico para o diagnóstico da doença. Portanto, o conhecimento das manifestações clínicas e de suas variações são os requisitos mais importantes para um diagnóstico acurado da doença (Ferraz, 2002).

Segundo a Associação Brasileira de Fisioterapia Neurofuncional (2021), a fisioterapia neurofuncional é área de especialidade da fisioterapia que atua nos comprometimentos resultantes de lesões no sistema nervoso, como no caso da doença de Parkinson. A fisioterapia neurofuncional, através do treinamento locomotor pode melhorar a marcha dos pacientes com doença de Parkinson (Suzuki \& De Carvalho, 2021). O treinamento locomotor promove melhora no padrão de marcha dos pacientes com doença de Parkinson, melhorando consequentemente a independência funcional.

A relevância desta pesquisa trata-se de compreender a relação da fisioterapia neurofuncional com a Doença de Parkinson. Neste sentido, devido à importância desta doença e da elevada prevalência de limitações locomotoras, julgou-se ser importante realizar um estudo abrangente dos cuidados da fisioterapia neurofuncional em pacientes com DP.

\section{Metodologia}

O trabalho visou a fazer uma revisão narrativa e atualização sobre a atuação da fisioterapia na Doença de Parkinson. Assim foram consultadas as bases de dados o Pubmed, o Scielo e o Lilacs com as palavras chaves Doença de Parkinson, Fisioterapia Neurofuncional e Doenças Neurológicas.

A pesquisa em questão construiu-se em oito etapas: elaboração da pergunta norteadora de investigação; produção de protocolo de investigação; elaboração de critérios de elegibilidade; estratégia de pesquisa e busca na literatura; coleta de dados; análise crítica dos estudos incluídos; extração de dados; discussão dos resultados e apresentação da revisão.

Para realizar este estudo, foram adotados os seguintes pontos de investigação:

a) em princípio, a partir de uma concepção bibliográfica do pressuposto na literatura sobre o tema em pauta, foram traçados os pontos mais relevantes, quanto ao que diz respeito à fisiopatologia, etiologia e diagnóstico da doença de Parkinson, e correlacionar com a atuação da fisioterapia. 
b) depois, pelas observações no desenvolvimento desta atividade, observou-se o que teóricos falam sobre a temática, traçando um diálogo entre eles.

A seleção das evidências científicas têm se dado a partir da adoção dos critérios de elegibilidade, sendo os critérios de inclusão:

a) textos completos disponíveis on-line;

b) estudos com desenhos experimentais, quase experimentais, observacionais e de revisão, sendo consideradas, também, pré-impressões;

c) recorte temporal de 2010 a fevereiro de 2021 ;

d) abordar a atuação da fisioterapia dentro do contexto de reabilitação e qualidade de vida de pacientes com a Doença de Parkinson.

E os critérios de exclusão:

a) estudos com pesquisas sem delineamento metodológico;

b) editoriais, comentários de especialistas, carta de editores e nota prévia;

c) trabalhos que não estejam enquadrados nos critérios de elegibilidade.

A pesquisa nas bases de dados eletrônicas foi realizada em setembro de 2021 com atualização em novembro de 2021.

\section{Resultados}

Dos 143 estudos encontrados mediante cruzamento dos descritores, 29 atenderam aos critérios de inclusão. Destes, 18 foram excluídos (11 repetidos, 4 não disponíveis na íntegra e 3 cujo resultado ainda não foi publicado), remanescendo 11 artigos que compuseram a amostra final (fluxograma 1). As principais informações dos artigos desta revisão estão apresentadas de maneira discursiva nos resultados. 
Fluxograma 1: mapeamento dos Estudos encontrados com os descritores.

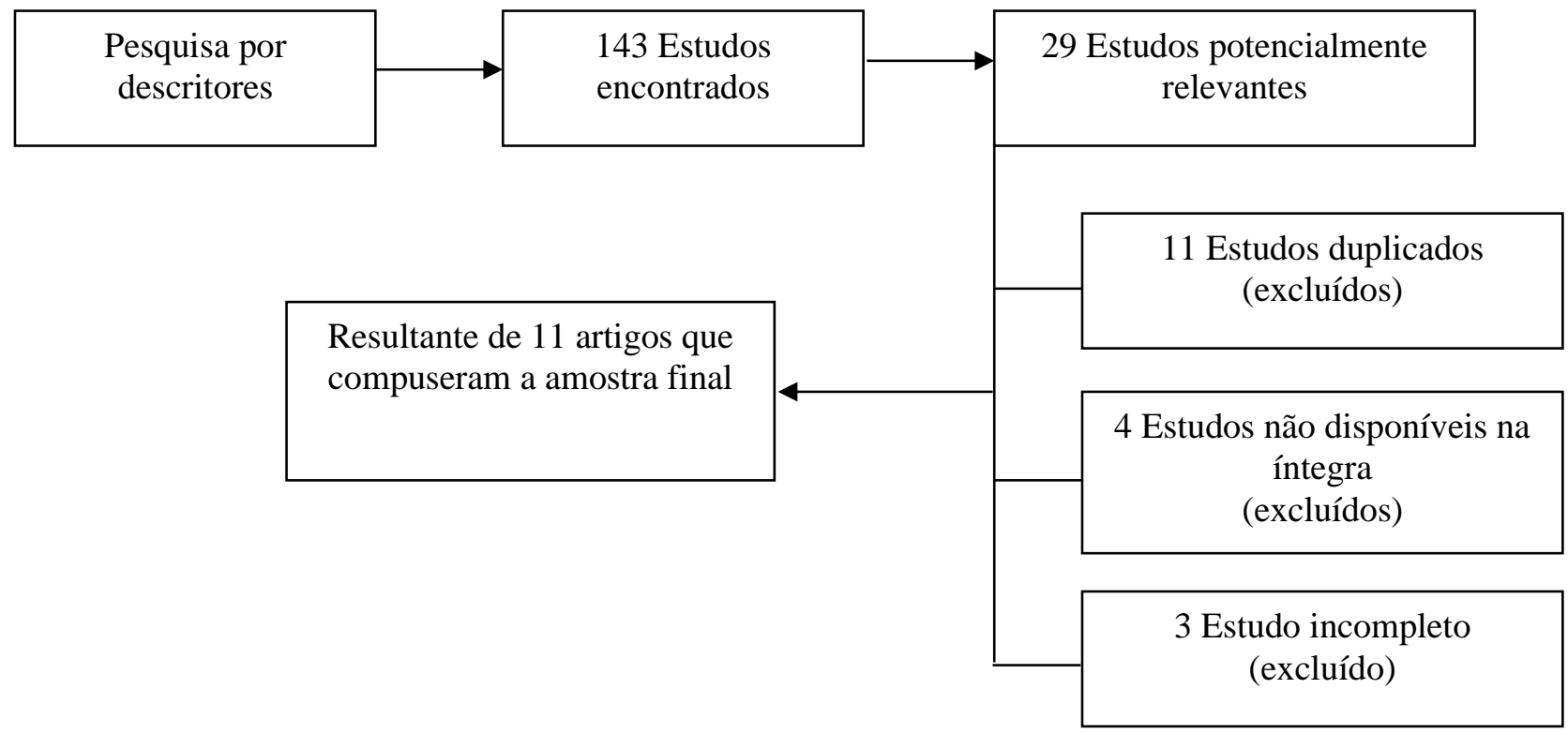

Fonte: Autores.

A fisioterapia é empregada como tratamento adjunto aos medicamentos ou a cirurgia utilizada na DP. De acordo com Dos Santos (2010) e seus colaboradores, a reabilitação deve compreender exercícios motores, treinamento de marcha (sem e com estímulos externos), treinamento das atividades diárias, terapia de relaxamento e exercícios respiratórios. Ainda segundo este estudo, outra meta é educar o paciente e a família sobre os benefícios da terapia por exercícios.

Segundo a Associação Brasil Parkinson (2007), a fisioterapia visa a uma reeducação e manutenção da atividade física, permitindo que o tratamento tenha uma melhor eficácia e, ainda, uma melhora psicológica do paciente portador de doença de Parkinson.

A intervenção fisioterápica inclui a terapia convencional e ocupacional, terapia com estímulos visuais, auditivos e somato-sensitivos. Os estímulos facilitariam os movimentos, o início e continuação da marcha, o aumento do tamanho dos passos e a redução da frequência e intensidade dos congelamentos (Nieuwboer A. et al.2001).

A fisioterapia voltada para pacientes parkinsonianos tem como objetivo minimizar os problemas motores causados tanto pelos sintomas primários da doença quanto pelos secundários, ajudando o paciente a manter a independência para realizar as atividades do dia-a-dia e melhorando sua qualidade de vida, que pode ser por uso de aparelhos auxiliares. (Greenberg et al., 2005). Tais aparelhos podem incluir corrimões adicionais colocados de forma estratégica pela casa para suporte adicional, talheres com cabos largos e toalhas de mesa antiderrapantes (Piemonte, 2003).

Com base nos achados da literatura que trouxe uma abordagem científica a respeito da temática, pode-se observar uma série de técnicas que possibilitam a melhora do paciente portador da Doença de Parkinson por meio da fisioterapia. Alguns autores elucidam seus resultados a partir da aplicação de testes, avaliações e técnicas terapêuticas.

Em estudo realizado, Cardoso e Pereira (2001) concluíram que a diminuição da amplitude torácica foi o fator determinante das alterações respiratórias restritivas dos parkinsonianos, limitando a elevação das estruturas do tórax e a expansibilidade pulmonar. Dessa forma, um programa de fisioterapia respiratória direcionado para o aumento da amplitude 
torácica promove a melhora da função respiratória e da capacidade funcional desses pacientes.

Para Cram (2002), um programa de fisioterapia personalizada para o paciente pode ajudar no controle dos problemas posturais, nas deformidades e distúrbios de marcha. Incluem-se no programa exercícios ativos e passivos, treinamento da caminhada, desenvolvimento de atividades diárias, calor, gelo, estimulação elétrica e hidroterapia.

No contexto da doença, a fisioterapia busca diminuir a disfunção física e permitir ao indivíduo realizar atividades do seu dia-a-dia com maior eficiência e independência possível. Para isso, o alongamento em pacientes com doença de Parkinson é necessário para a melhora de amplitude de movimento (Dias et al., 2005).

No estudo de Morries (2006) teve como objetivo de considerar o papel do fisioterapeuta no treinamento motor de portadores da doença de Parkinson. E ao final do estudo viu-se que parte do programa de treinamento teve participação do fisioterapeuta com intuito de otimizar a qualidade de vida e a vida social.

Johnson (2007) visou estudar o uso de exercícios motores na doença de Parkinson. Concluiu que os portadores de doença de Parkinson devem ser incentivados a manter uma atividade física juntamente com o tratamento farmacológico. Braga (2003) Mostrou que a reabilitação da marcha e equilíbrio do doente de Parkinson requer vários recursos e abordagens. E concluiu que exercícios resistidos devem ser aplicados no tratamento dos portadores de doença de Parkinson.

Goodwin (2008) revisou sistematicamente ensaios clínicos randomizados sobre a efetividade das intervenções sobre os resultados do exercício para pessoas com doença de Parkinson. E foi evidenciado os benefícios do exercício para portadores da doença de Parkinson.

King (2009) desenvolveu um programa de exercícios para minimizar a incapacidade funcional em pessoas com doença de Parkinson. Um programa de mobilidade, como o apresentado neste artigo, teria de ser sustentada e modificados ao longo do curso da doença para manter o benefício máximo.

Pelosin (2009) avaliou os efeitos do treinamento na esteira e cicloergômetro. O treinamento melhorou significativamente o desempenho da caminhada. Stanley (2000) avaliar e comparar a função cardiopulmonar de indivíduos com doença de Parkinson com a de indivíduos saudáveis. Os indivíduos com doença de Parkinson foram menos eficientes durante o exercício.

Com a progressão da doença, a coordenação motora fica comprometida e o doente diminui suas atividades diárias, desencadeando uma atrofia muscular. Com o exercício, o aumento da mobilidade pode de fato modificar a progressão da doença e impedir contraturas, além de ajudar a retardar a demência (Braga et al., 2002).

Por outro lado, exercícios não impedem a progressão da doença, mas mantêm um estado de funcionamento muscular e osteoarticular conveniente. Anos de evolução de rigidez e bradicinesia produzem alterações patológicas ósseas (osteoporose e artrose) responsáveis por uma incapacidade funcional ainda mais limitante. Além disso, o bom impacto dos exercícios sobre a disposição e o humor são pontos favoráveis a esta terapia (Bittencourt, 2003).

\section{Considerações Finais}

O presente estudo avaliou a importância da atuação da fisioterapia na doença de Parkinson, buscando traçar os principais pontos das intervenções realizadas por estes profissionais. Todos os objetivos deste trabalho foram alcançados, chegando a uma conclusão de que há uma grande necessidade para a melhoria na reabilitação e qualidade de vida em pacientes com a doença de Parkinson. 
Essa revisão bibliográfica mostrou que há benefícios de todas as intervenções relacionadas à fisioterapia convencional e também com a fisioterapia neurológica. Mas como houve uma dificuldade encontrar literaturas disponíveis sobre o assunto, fica a sugestão para que sejam realizados estudos que avaliem a eficácia desse método e que possuem uma amostra razoável.

Neste pensar, pode-se concluir que o fisioterapeuta pode utilizar os mais diversos tipos de tratamento que estão incluídos na fisioterapia neurofuncional, pois é considerado como um método seguro, eficaz e acessível como instrumento qualitativo de assistência.

\section{Referências}

ABRAFIN. Associação Brasileira de Fisioterapia Neurofuncional. https://abrafin.org.br

Araki, I, \& Kuno S. (2000) Assessment of Voiding dysfunction in Parkinson's disease by the international prostate symptom score. J Neurol Neurosug Psychiatry. 68: 429-33.

Bittencourt, P. C. T. et al. (2003) Doença de Parkinson: diagnóstico e tratamento.

Braga, A, Xavier, A. L., \& Machado, R. P. (2002) Benefícios do treinamento resistido na reabilitação da marcha e equilíbrio nos portadores da doença de Parkinson. Revista da Pós-Graduação da Universidade Gama Filho.

Brasil. Ministério da Saúde, Secretaria da Atenção à Saúde. Portaria n 228, de 10 de maio de 2010. Protocolo clínico de diretrizes terapêuticas - doença de Parkinson.

Cardoso, S. R., \& Pereira, J. S. (2001) Análise funcional da complacência torácica na doença de Parkinson. Fisioterapia Brasil, 2(1), 41-46.

Cram, D. L. (2002) Entendendo a síndrome de Parkinson. Ciência Moderna.

Da Costa Silva, P. F., et al. (2011) Correlação entre perfil clínico, qualidade de vida e incapacidade dos pacientes da Associação Brasil Parkinson. ConScientiae Saúde, 10(4).

Dias, N. P., Fraga, D. A., Cacho, E. W. A., \& Oberg, T. D. (2005) Treino de marcha com pistas visuais no paciente com doença de Parkinson. Fisioterapia em Movimento. (4).

Dos Santos, V. V. et al. (2010) Fisioterapia na doença de Parkinson: uma breve revisão. Rev. bras neurol, 46(2), 17-25.

Ferraz, H. B., \& Borges, V. (2002) Doença de parkinson. Revista brasileira de medicina, 59(4), 207-219.

Ferreira, M. G. S. Exercício físico com pessoas com doença de Parkinson. Tese de Doutorado.

Goodwin, V. A., Richards, S. H., Taylor, R. S., Taylor, A. H., \& Campbell, J. L. (2008) The effectiveness of exercise interventions for people with Parkinson disease: a systematic review and metaanalysis. Mov Disord.

Greenberg, D. A., Aminoff, M. J., \& Simon, R. P. Neurologia clínica. (5a ed.), Artmed.

Hauser, R. A., \& Zesiewicz, T. A. (2001) A Doença de Parkinson: Perguntas e Respostas. Spain. 3ª.Edição. Atlas Medical Publishing Ltda. $37-43$.

Johnson, A. M., \& Almeida, Q. J. (2007) The impacto of exercise rehabilitation and physical activityon the management of Parkinson`s disease. Geriatrics \& Aging.

King, L. A., \& Fay, B. H. (2009) Delaying Mobility Disability in People With Parkinson Disease Using a Sensorimotor Agility Exercise Program. Journal of the American Physical Therapy Association

Ministério da Saúde, Conselho Nacional de Saúde, Comissão Nacional de ética em Pesquisa. Resolução Nº 196/96 versão 2012.

Morris, M. E. (2006) Locomotor Training in People With Parkinson Disease. Journal of theAmerican Physical Therapy Association.

Navarro, F. M., Marcon, S. S., \& Teston, É. F. (2015) Descobrindo adoença de parkinson: impacto para o parkinsoniano e seu familiar. Ciência, Cuidado e Saúde, 7.

Nieuwboer A et al. (2001) The effect of a home physiotherapy program for persons with Parkinson'sdisease. J Rehab Med.

Parkinson, J. (1887) An Essay on the Shaking Palsy. Whittingham and Rowland.

Pelosin, E, et al. (2009) Effects of treadmill training on walking economy in Parkinson'sdisease: apilot study. Neurol Sci.

Pereira D, \& Garrett C. (2010) Factores de risco da doença de Parkinson um estudo epidemiológico.Acta Med Port.

Piemonte, M. E. P. (2003) Programa semanal de exercícios para pacientes com doença deParkinson. Lemos. 
Research, Society and Development, v. 11, n. 2, e5211225363, 2022

(CC BY 4.0) | ISSN 2525-3409 | DOI: http://dx.doi.org/10.33448/rsd-v11i2.25363

Pieruccini-Faria, F. et al. (2006) Parâmetros cinemáticos da marcha com obstáculos em idosos comdoença de Parkinson, com e sem efeito da levodopa: um estudo piloto. Brazilian Journal of Physical Therapy, 233-239.

Sammour, M. Z. (2007) Distúrbios miccionais em pacientes com doença de Parkinson: associaçãoentre parâmetros clínicos e urodinâmicos. Faculdade de Medicina de São Paulo.

Souza, R. N. (2002) Análise das disfunções urinárias na doença de Parkinson. Universidade Estadualde Campinas, SP.

Suzuki, T. S., \& De Carvalho, M. P. (2018) Fisioterapia Neurofuncional naDoença de Parkinson.

Teive, A. G. H. . (1998) Sielo Brasil: O papel de charcot na doença de Parkinson. Arq. Nero-Psiquiatr56(1):4-14.

Teive, H. A. G. . 2003) Etiopatogenia da doença de Parkinson. Doença de Parkinson. Guanabara Koogan, 33-37. 